\title{
Weak Lensing of the CMB by Large Scale Structure
}

\author{
A. Amblard ${ }^{1}$, C. Vale $^{2}$ \\ and M. White ${ }^{1,2}$ \\ 1, University of California, Berkeley, department of Astronomy, Berkeley CA 94720, USA \\ email: amblard@astro.berkeley.edu \\ ${ }^{2}$ University of California, Berkeley, department of Physics, Berkeley CA 94720, USA
}

\begin{abstract}
Several recent papers have studied lensing of the CMB by large-scale structures, which probes the projected matter distribution from $z=10^{3}$ to $z \simeq 0$. This interest is motivated in part by upcoming high resolution, high sensitivity CMB experiments, such as APEX/SZ, ACT, SPT or Planck, which should be sensitive to lensing. In this paper we examine the reconstruction of the large-scale dark matter distribution from lensed CMB temperature anisotropies. We go beyond previous work in using numerical simulations to include higher order, non-Gaussian effects and find that the convergence and its power spectrum are biased, with the bias increasing with the angular resolution. We also study the contamination by the kinetic Sunyaev-Zel'dovich signal, which is spectrally indistinguishable from lensed CMB anisotropies, and find that it leads to an overestimate of the convergence. We finish by estimating the sensitivity of the previously cited experiments and find that all of them could detect the lensing effect, but would be biased at around the $10 \%$ level.
\end{abstract}

\section{Introduction}

Weak gravitational lensing recently became a powerfull tool to map the mass distribution in the Universe, allowing to constrain cosmological parameters and testing our paradigm of structure formation (see Van Waerbeke \& Mellier (2003) for a recent review). Furthermore another observational window is about to open with the future millimeter surveys such as ACT, APEX-SZ, Planck and SPT. These surveys will measure the Cosmic Microwave Background (CMB) fluctuations emitted around $z \sim 10^{3}$ and could probe the photon deflection induced by large-scale mass distribution as they journey to our present time. A precise measurement of those deflections could put very strong contrains on cosmological parameters, such as the neutrino mass and the dark energy equation of state (Kaplinghat et al. (2003)).

Since the pionner work of Zaldarriaga \& Seljak (1999) a considerable effort (Hirata \& Seljak (2003b), Kesden et al. (2003), Okamoto \& Hu (2003)) has been put into developing an accurate estimator of this effect. The most recent estimators can be separate into 2 kinds : maximum likelihood estimator (Hirata \& Seljak (2003a)) and quadratic estimator $(\mathrm{Hu}(2001 \mathrm{a}))$. Both look for non-Gaussianity produced by the projected large scale structure density as it lenses the CMB photons, they both assume the absence of foreground (or perfect component separation) and the Gaussianity of the CMB and large scale matter distribution fields.

In this work, we tried to move a step further, and test 2 previous assumptions against 2 observationally irreducible complications : the non-Gaussianity of matter distribution and the kinetic Sunyaev-Zel'dovich (kSZ) effect (Sunyaev \& Zel'dovich (1972), Sunyaev \& Zel'dovich (1980); for recent reviews Rephaeli (1995), Birkinshaw (1999), Carlstrom 
et al. (2002)). We have elected to use the quadratic estimator for our reconstructions as it is computationnally less involving and roughly equivalent Hirata \& Seljak (2003a).

\section{Weak lensing of the CMB \& quadratic estimator}

In the weak lensing limit (small deflection angle), the gravitational lensing of the CMB simply remaps the primary temperature field Seljak (1996) :

$$
T(\boldsymbol{\theta})=\tilde{T}\left(\boldsymbol{\theta}^{\prime}\right)=\tilde{T}(\boldsymbol{\theta}-\boldsymbol{\delta} \boldsymbol{\theta})=\tilde{T}(\boldsymbol{\theta})-\boldsymbol{\nabla} \tilde{T}(\boldsymbol{\theta}) . \boldsymbol{\delta} \boldsymbol{\theta}
$$

where $T(\boldsymbol{\theta})$ is the observed temperature at position $\boldsymbol{\theta}$ (boldface represents vector), $\tilde{T}\left(\boldsymbol{\theta}^{\prime}\right)$ is the unlensed temperature at the position $\boldsymbol{\theta}^{\prime}$ and $\boldsymbol{\delta} \boldsymbol{\theta}$ is the deflection angle. For small $\boldsymbol{\delta} \boldsymbol{\theta}$, the lensing effect is then just expressed as the product of the unlensed CMB gradient with the deflection angle. In that case, one can build an optimal quadratic estimator $(\mathrm{Hu}$ (2001b), Cooray \& Kesden (2003)) of the convergence $\kappa_{\text {est }}$.

$$
\kappa_{\mathrm{est}}(\ell)=\frac{A_{\ell}}{2} \int \frac{d^{2} \ell_{1}}{(2 \pi)^{2}} F\left(\boldsymbol{\ell}_{\mathbf{1}}, \ell_{\mathbf{2}}\right) T\left(\boldsymbol{\ell}_{\mathbf{1}}\right) T\left(\boldsymbol{\ell}_{\mathbf{2}}\right)
$$

with $\kappa_{\text {est }}$ being the estimated convergence map (related to the deflection angle by $\boldsymbol{\nabla} \boldsymbol{\delta} \boldsymbol{\theta}=$ $2 \kappa), \boldsymbol{\ell} \equiv \boldsymbol{\ell}_{\mathbf{1}}+\boldsymbol{\ell}_{\mathbf{2}}$, and where :

$$
F\left(\ell_{1}, \ell_{2}\right) \equiv \frac{\ell \cdot\left(\ell_{1} \tilde{C_{\ell_{1}}}+\ell_{2} \tilde{C_{\ell_{2}}}\right)}{2 C_{\ell_{1}}^{\text {tot }} C_{\ell_{2}}^{\text {tot }}} \quad A_{\ell}^{-1}=\ell^{2} \int \frac{d^{2} \ell_{1}}{(2 \pi)^{2}} 2 C_{\ell_{1}}^{\text {tot }} C_{\ell_{2}}^{\text {tot }} F^{2}\left(\ell_{1}, \ell_{2}\right)
$$

This convergence, which is the variable we use to describe the lensing, represents in fact the integrated matter distribution along the line of sight. (multiply by some geometrical factors). In order to determine if the map is correctly reconstructed we use the crossspectrum between the estimated map and the input one :

$$
\left\langle\kappa(\ell) \kappa_{\text {est }}^{*}\left(\ell^{\prime}\right)\right\rangle=2 \pi \delta\left(\ell-\ell^{\prime}\right) C_{\ell}^{c r o s s}
$$

This cross-spectrum $C_{\ell}^{\text {cross }}$ should be equal to the input convergence power spectrum $C_{\ell}^{\kappa \kappa}$ if the map is not biased. Apart from the map, we also want to reconstruct the convergence power spectrum which can be expressed as :

$$
\left\langle\kappa_{\text {est }}(\ell) \kappa_{\text {est }}^{*}\left(\ell^{\prime}\right)\right\rangle=2 \pi \delta\left(\ell-\ell^{\prime}\right) C_{\ell}^{\text {auto }}=2 \pi \delta\left(\ell-\ell^{\prime}\right)\left(C_{\ell}^{\text {est }}+\mathcal{N}_{\ell}^{\text {est }}\right)
$$

where $\left(C_{\ell}^{\text {est }}\right.$ is the estimate of $C_{\ell}^{\kappa \kappa}$ and $\mathcal{N}_{\ell}^{e s t}$ is the noise power in the map. This noise power is expressed as $\mathcal{N}_{\ell}^{\text {est }}=\frac{\ell^{2}}{4}\left(A_{\ell}+A_{\ell}^{N G}+\cdots\right)$ (Cooray \& Kesden (2003)), where $A_{\ell}$ is our convergence map normalization factor and $A_{\ell}^{N G}$ represents an higher order term (in $\kappa^{2}$ ). The latter requires $C_{\ell}^{\kappa \kappa}$ in order to be computed, so one need realistically to iterate the convergence power spectrum estimation, however here we choose to suppose this correction known (i.e. that such an algorithm converge perfectly). 


\section{Simulations}

Our test simulations consist of a $30 \times 30$ degree field with a $2 \mu \mathrm{K} /$ arcminute instrumental Gaussian white noise, we generate a CMB map using the flat sky approximation. The convergence map are created as follow :

- for the Gaussian case : a Gaussian random field realization is generated with a power spectrum computed using Limber's approximation;

- for non-Gaussian case : the convergence map is a mixture of Gaussian field for high redshift ( $z>2$ computed as in the previous case) and non-Gaussian field for low redshift coming from a N-body simulations (TreePM code from White (2002) with a $300 h^{-1} \mathrm{Mpc}$ side size and a $512^{3}$ number of particules, see Schulz \& White (2002) and Vale et al. (2004) for details).

The convergence simulations are $7.5 \times 7.5$ degrees wide (size limit come from N-body simulation) and are flipped to fill the $30 \times 30$ degree field and avoid discontinuity. Thermal and kinetic SZ maps are created from the N-body simulations and we assume the baryons trace the dark matter and the isothermality of the clusters.

\section{Gaussian versus Non-Gaussian}

We reconstruct the convergence map with the quadratic estimator of $\mathrm{Hu}$ (2001a) as indicated in section 2 for the Gaussian and non-Gaussian simulations.
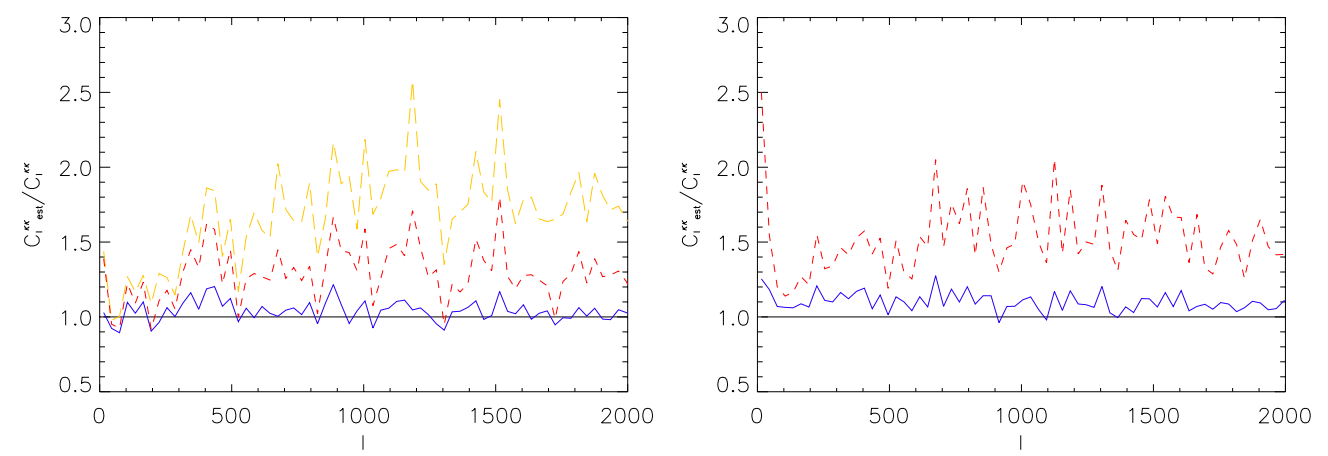

Figure 1. $C_{\ell}^{\text {cross }} / C_{\ell}^{\kappa \kappa}$ (blue solid line) showing the estimated map bias, $C_{\ell}^{\text {est }} / C_{\ell}^{\kappa \kappa}$ with a $0^{\text {th }}$ order (in $\kappa$ ) noise correction (orange long dashed line), and $C_{\ell}^{e s t} / C_{\ell}^{\kappa \kappa}$ with a $2^{\text {nd }}$ order (in $\kappa$ ) noise correction (red dashed line) showing estimated convergence power spectrum bias (Gaussian case on the left, non-Gaussian case on the right).

We found that the reconstructed map is biased in the non-Gaussian case as indicated in Figure 1 (blue solid curve) by 10\%. Furthermore the estimated power spectrum is biased in both cases. In the Gaussian case, the power spectrum is biased by $25 \%$ due to term in $\kappa^{4}$ not included in the noise estimate. In the non-Gaussian case, the power spectrum is biased by $50 \%$, the additionnal $25 \%$ coming from non-Gaussian feature in the $\kappa$ field.

\section{Kinetic SZ contamination}

We add the kinetic SZ as a contaminant, and reconstruct our maps in 2 cases : one where we just add the kSZ power spectrum as an extra noise term and one where in 
addition we mask out the clusters (using the thermal SZ simulation with a $50 \mu \mathrm{K}$ cutoff value, $1.4 \%$ of the pixels).

The bias of the estimated convergence jump up to $20 \%$ (figure 2, blue solid line) if we do not mask out clusters, fortunately the masking procedure reduces to a negligeable amount the correlated features of the lensing and $\mathrm{kSZ}$.
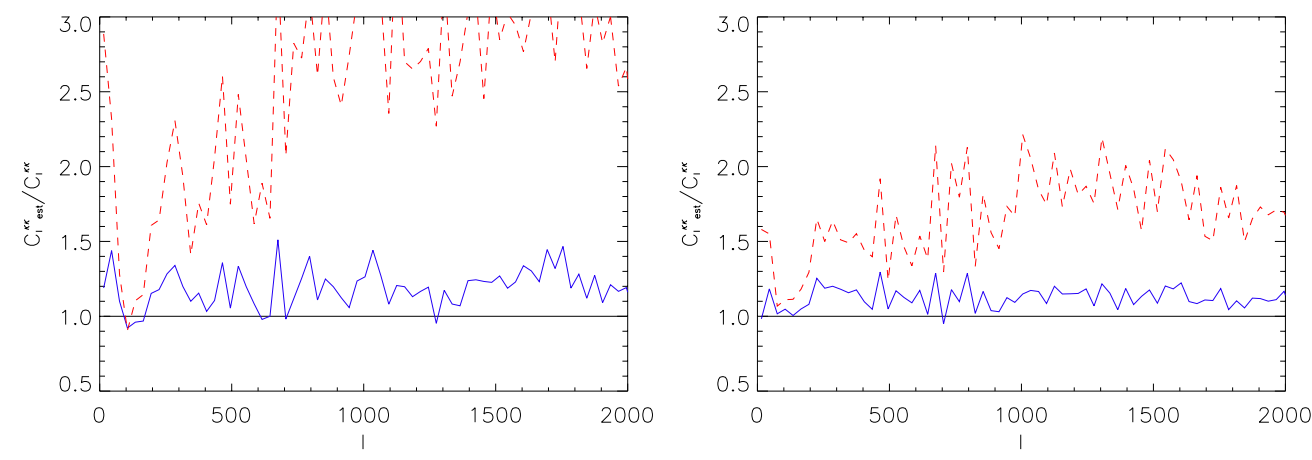

Figure 2. $C_{\ell}^{\text {cross }} / C_{\ell}^{\kappa \kappa}$ (solid line) showing the estimated map bias, and $C_{\ell}^{\text {est }} / C_{\ell}^{\kappa \kappa}$ with a $2^{\text {nd }}$ order (in $\kappa$ ) correction (dashed line) showing convergence power spectrum bias (kSZ unmasked on the left and masked on the right).

The power spectrum estimate is highly biased when we do not mask the clusters (200\%) but is reduced to $70 \%$ with our masking technique (only $20 \%$ additionnal to non-Gaussian effect). We conclude that even if the kSZ is locally stronger than the lensing, its contamination can be controlled by masking clusters area (note that our masking is in no way optimal, so that one can hope to get down to even lower residual).

\section{Bias and forecasting experiment results}

Our final step is to consider how the resolution of the experiment would modify our result.
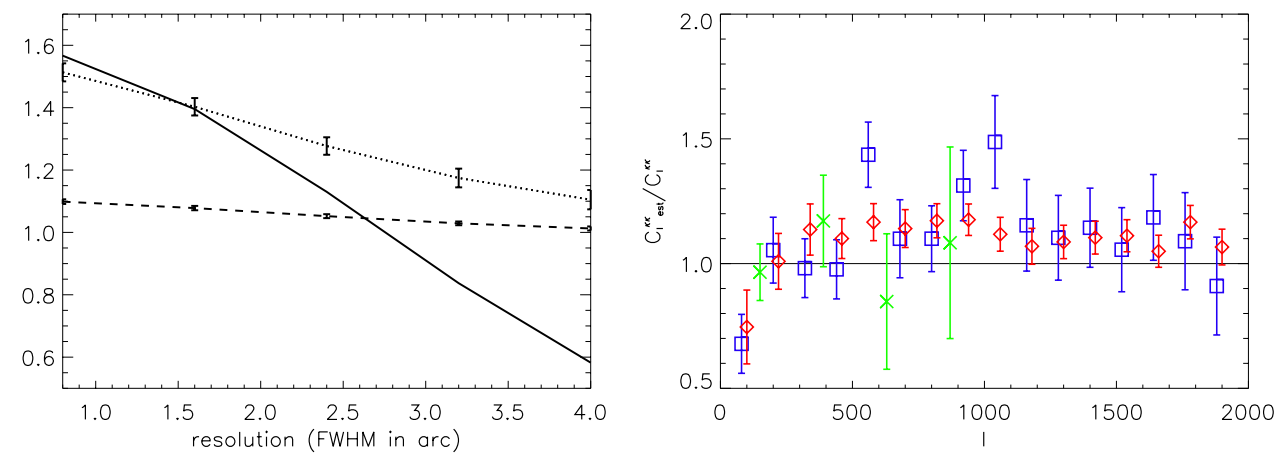

Figure 3. Biases (dashed line for map one and dotted line for power spectrum one) and signal-to-noise ratio (solid line) versus resolution on the left, precision and bias of the recovered $\kappa$ power spectrum $\left(C_{\ell}^{e s t} / C_{\ell}^{\kappa \kappa}\right)$ for 3 forthcoming surveys (squares : APEX-SZ, diamonds : SPT, crosses : $1 / 10^{\text {th }}$ of Planck) on the right. 
The resolution is an important factor as all the bias we are studying are coming from small structures, therefore suppressing those structures by smoothing on larger scale can reduce these biases. We compute the signal-to-noise ratio and the 2 biases ( $\kappa$ map and $\kappa$ power spectrum ones) averaging over structures from mode 0 to 2000. As expected the biases reduce as the beam size increases (figure 3), but the signal-to-noise ratio is decreasing too. Therefore, an experiment which favours the sky coverage against the angular resolution and the noise level may be more suitable to get a precise measurement of the CMB lensing. Figure 3 shows also the precision and bias forecast for 3 different experiments : APEX-SZ, SPT, and Planck (we took only $1 / 10^{\text {th }}$ of Planck survey to stay in the flat sky approximation). SPT and APEX-SZ which have a greater angular resolution than Planck are biased by 10\%, Planck power spectrum estimate is unbiased at our level of precision. The respective error bars (average over 125 modes for APEX-SZ and SPT, 250 modes for Planck) of these experiments are around 7, 15, $30 \%$ for SPT, APEX-SZ and $1 / 10^{\text {th }}$ of Planck. As shown by the errobars on figure 3 , the CMB lensing is clearly detectable by these 3 surveys, and APEX-SZ might well be the first experiment to detect it.

\section{Conclusions}

The CMB lensing is a promising tool to probe our cosmological paradigm and cosmological parameter values. However to obtain constraining results, one need to measure it with a very good accuracy (typically a few percent). We show that our precision will be limited by bias coming from the non-Gaussianity of the dark matter distribution, and by the contamination of the kinetic SZ. Despite this limitation, forthcoming surveys should detect the CMB lensing. The next generation of surveys with polarization capability may be more suitable to get over the kSZ limitation but would have to deal with non-Gaussianity.

\section{References}

Amblard, A., Vale, C. \& White, M. 2004, to be published in NewA, preprint astro-ph/0403075 Birkinshaw M. 1999, Phys. Rep. 310, 98

Carlstrom, J., Holder, G. \& Reese, E. 2002, ARAA 40, 643

Cooray, A. \& Kesden, M. 2003, NewA 8, 231

Hirata, C.M. \& Seljak, U. 2003a, Phys. Rev. D 67, 043001

Hirata, C.M. \& Seljak, U. 2003b, Phys. Rev. D 68, 083002

Hu, W. 2001a, ApJ 557, L79

Hu, W. 2001b, Phys. Rev. D 64, 083005

Kaplinghat, M., Knox, L. \& Song, Y.-S. 2003, Phys. Rev. Lett. 91, 241301

Kesden, M., Cooray, A. \& Kamionkowski, M. 2003, Phys. Rev. D 67, 123507

Okamoto, T. \& Hu, W. 2003, Phys. Rev. D 67, 083002

Rephaeli, Y. 1995, ARAESA 33, 541

Schulz, A.E. \& White, M. 2003, ApJ 586, 723

Seljak, U. 1996, ApJ 463, 1

Sunyaev, R.A. \& Zel'dovich Y.B. 1972, Comm. Astrophys. Space Phys. 4, 173

Sunyaev, R.A. \& Zel'dovich Y.B. 1980, ARAEA A 18, 537

Vale, C., Amblard, A. \& White, M. 2004, to be published in NewA, preprint astro-ph/0402004

Van Waerbeke, L. \& Mellier, Y. 2003, preprint astro-ph/0305089,

White, M. 2002, ApJS 143, 241

Zaldarriaga, M. \& Seljak, U. 1999, Phys. Rev. D 59, 123507 